\title{
Time-frequency Analysis of Output Power of Wind Turbine Based on Wavelet Transform
}

\author{
Hao Guo ${ }^{\mathrm{a}}$, Zheng $\mathrm{Li}^{\mathrm{b}}$ \\ College of Information Science and Technology, Donghua University, Shanghai China, 201620 \\ a'Guohaogoodluck@163.com, blizheng@dhu.edu.cn
}

\begin{abstract}
Instability is one of shortcomings for wind power. Along with the increasing of the number of wind farms, power instability also increased. The fluctuation of wind power will cause the variation of voltage in access point .The impact of this fluctuation to power grid is more and more bigger. To reduce this impact, we should smooth and filter the output power. Therefore, a detailed analysis of the characteristics of output power is very necessary. This paper starts with the study of stationary principle. Then study second-time-scale output power. We find the power is non-stationary random signal sequence. This sequence does not have the concept of spectrum, so we choose wavelet analysis, which is commonly used in non-stationary random signal analysis, to do the time-frequency analysis of the output power.
\end{abstract}

Keywords-output power of wind turbine, stationary test, wavelet analysis

\section{INTRODUCTION}

The random fluctuation of wind determines the fluctuation of output power, which will impact the security and stability of power system. With the increase of capacity of wind turbine connected to the grid, the impact on the grid is paid more and more attention. Seen from the grid side, wind farms connected to the grid is equivalent to a disturbance source with stochastic properties, the impact to the quality and stability of the power system can not be ignored. Therefore, analysis of characteristic of output power is the basis of the problem.

In this field, many foreign and domestic scholars have carried out some research ${ }^{[1,2,3]}$. Paper [4] researched the characteristics of output power of wind farm and wind farm group, compared the different characteristics of different timescales of wind output power. Paper [5] researched the statistical rules of wind power output fluctuation in different space-time scale from correlation and smoothness aspects. At the same time, do the hypothesis and verification of wind power fluctuation probability distribution model based on sampling data. Paper [6] realized that it is difficult to describe the generation law of power fluctuation from the angle of time domain, then studied from the angle of frequency domains.

However, there is no one analysis the output characteristics from the output series stationary and application of wavelet transform aspects, this article focuses on these aspects.

\section{CHARACTERISTICS OF STATIONARY SEQUENCE}

Stationary random sequence has two characteristics:

a. The mean of the sequence is constant;

b. The autocorrelation function is only related to the time interval. But it has nothing to do with the starting point of the sequence.

The corresponding mathematical expressions is:

(1) The mean function

$$
u_{t}=E\left(X_{t}\right)=\int_{-a}^{a} X d F_{t}\left(X_{t}\right)
$$

Where, $u_{t}$ is the mean function of the sequence $\left\{X_{t}\right\}$

(2) The autocorrelation functions

$$
\rho(t, s)=\frac{r(t, s)}{\sqrt{r(t, t) r(s, s)}}
$$

Where, $r(\mathrm{t})$ is the relation function of the sequence $\left\{X_{t}\right\}$

\section{SimUlation ANALYSIS OF Stationary OF OUTPUT POWER}

\section{A. Data description}

The data used in the study is shown in Fig1.The capacity of wind turbine is 2MW. We sampled every second power in one hours from the wind turbine.

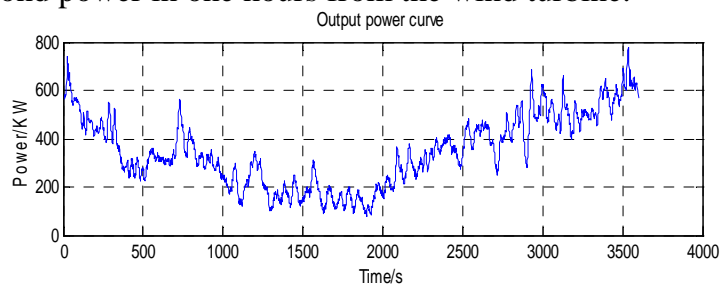

Figure 1. Output power curve

\section{B. Mean analysis}

Calculate the average value in 5 and 20 seconds .The results are shown in Fig2; 3. 


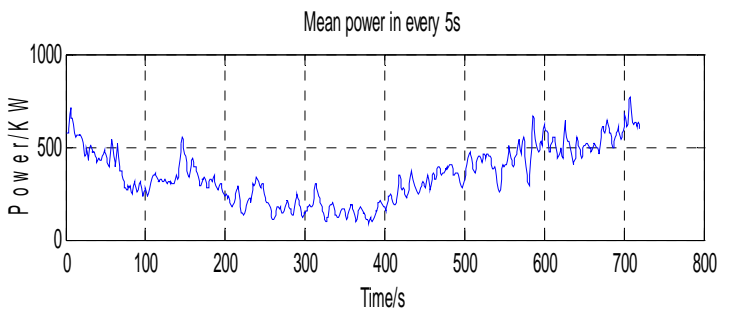

Figure 2. Mean power in 5 seconds

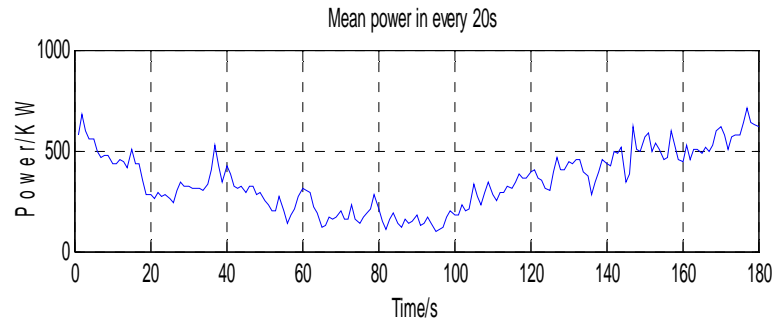

Figure 3. Mean power in 20 seconds

We can see, the average values of power are not constant in both every 5 seconds and 20 seconds, so the output power is not stationary.

\section{Autocorrelation analysis}

Do the correlation analysis with the output power in Matlab. We get the Fig4.

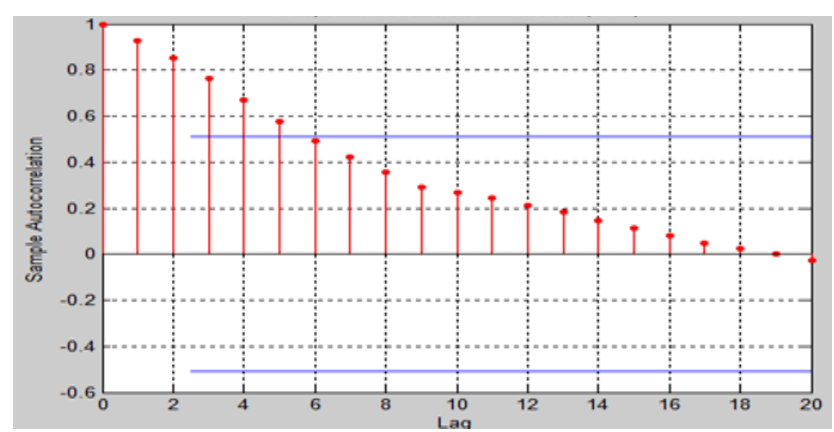

Figure 4. Autocorrelation analysis result

We can see, the points do not change in the vicinity of 0 . So we know the output power is not stationary sequence.

Based on the two aspects analysis results above, we can draw a conclusion that the output power of the second series is non-stationary series.

If we still research from the frequency angle, we can use the Sptool toolbox to do spectrum analysis. The results is shown in Fig5.

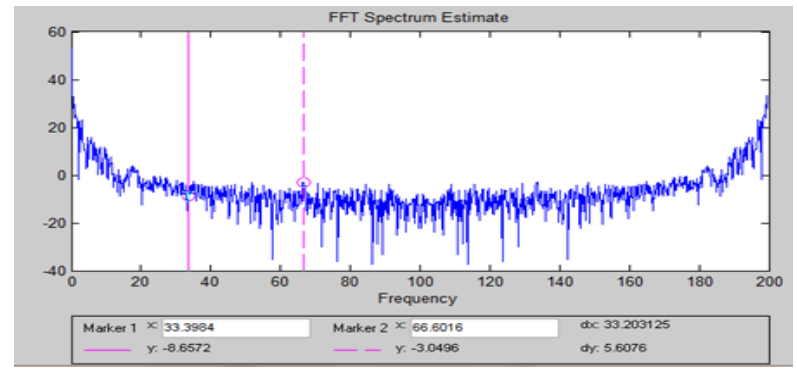

Figure 5. Spectrum analysis result( $\mathrm{Y}$-axis is energy at a certain frequency.)

It can be seen from the figure, from 0 to $200 \mathrm{~Hz}$ frequency band, the energy value basically is lower than 0 .So we can't get the main components of the power. This angle doesn't work.

\section{PRINCIPLE OF WAVELET ANALysis}

As above, we know that the output power series is non-stationary random sequence. The most commonly used tool of non-stationary signal is wavelet transform analysis.

Wavelet is the small wave ${ }^{[7]}$. The so-called "small" means the decay of weak; while the "wave" refers to its volatility. Compared with the Fourier transform, the wavelet transform is a time frequency localization analysis (spatial).It can automatically adapt to the time-frequency signal analysis requirements, which can focus on any signal details to solve the difficult problem.

\section{V.WAVELET ANALYSIS RESULT}

\section{A. Wavelet time-frequency analysis}

Do the wavelet time-frequency analysis. The result is shown in Fig6:

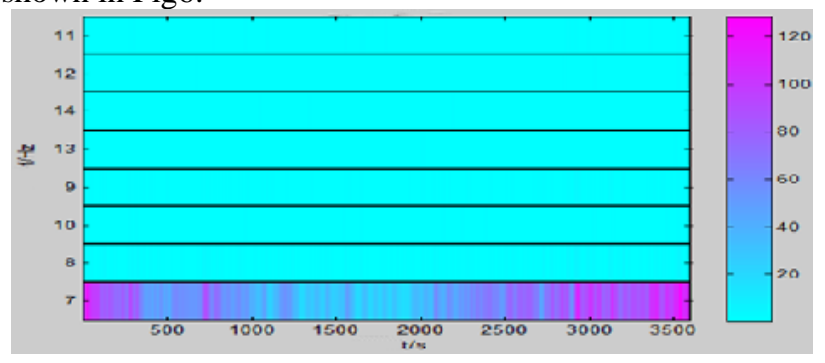

Figure 6. Wavelet time-frequency analysis result

From Fig6, we know the main frequency component of the second level output power is from $7.5 \mathrm{~Hz}$ to $13.5 \mathrm{~Hz}$. The highest energy frequency components near the $7.5 \mathrm{~Hz}$, with the energy amplitude of individual points as high as 120 .

\section{B. One-dimensional discrete wavelet analysis}

We use the wavelet toolbox provided by Matlab do the single-scale and multi-scale decomposition for the output power. The results are as follows: 


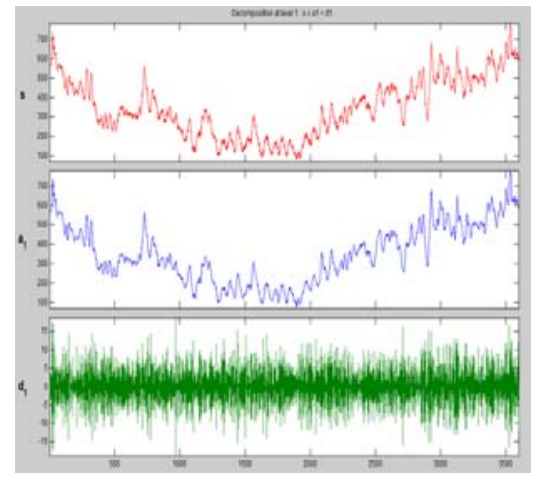

Figure 7. Single-scale decomposition result

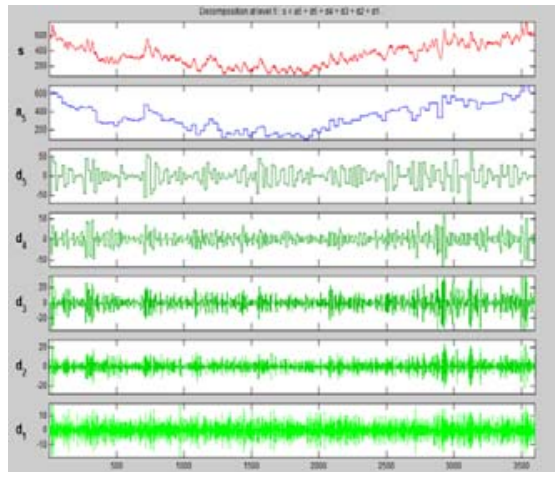

Figure 8. Multi-scale decomposition result (Level=5)

In the figure, $S$ is the ordinary signal, $a$ is the approximation of the ordinary signal, $d$ is the details signal of the ordinary signal (or $a$ is high frequency signal, $d$ is the low-frequency signals)

We can learn: approximate signals could reflect the characteristics of the original signal to a great extent, the detail signal work only as supplement. Besides, we can find periodic component in the low frequency signal.

\section{Wavelet packet decomposition}

Do the three layers decomposition based on $\mathrm{db} 1$ wavelet packet on the output power.

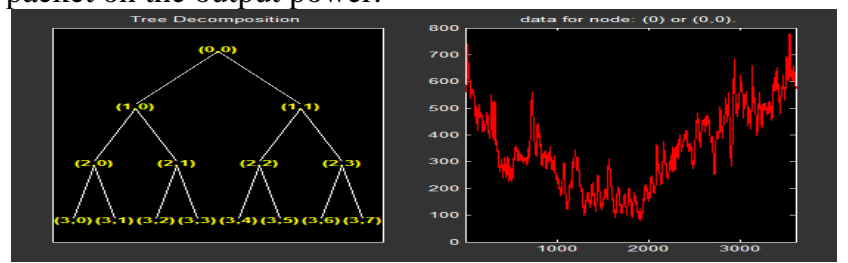

Figure 9. Three layers decomposition based on db1 wavelet packet

From Fig8, we can know all components in different levels of the output power.

\section{Wavelet denoising}

First denoising in default threshold, find the result is not well, then denoising in adjusted threshold The result is shown in Fig10
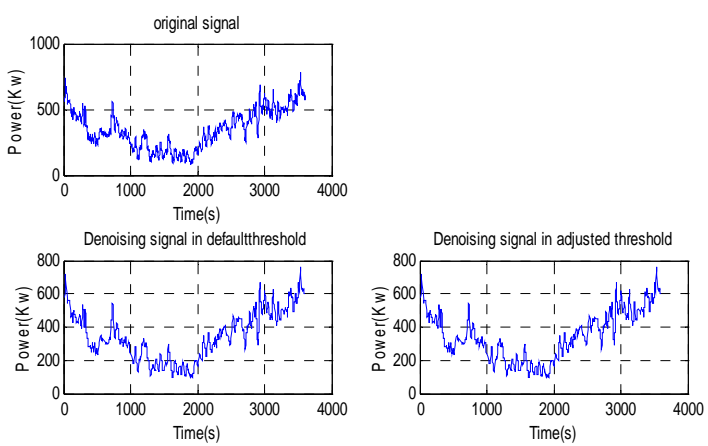

Figure 10. Wavelet denoising results

We learn that wavelet denoising can smooth the power. And adjusted threshold denoising is better than default threshold denoising.

\section{E. Contrastive study before and after denoising}

Do the three layers decomposition based on db1 wavelet packet on the Denoising signal in adjusted threshold. The results are shown in Fig11 and Fig12
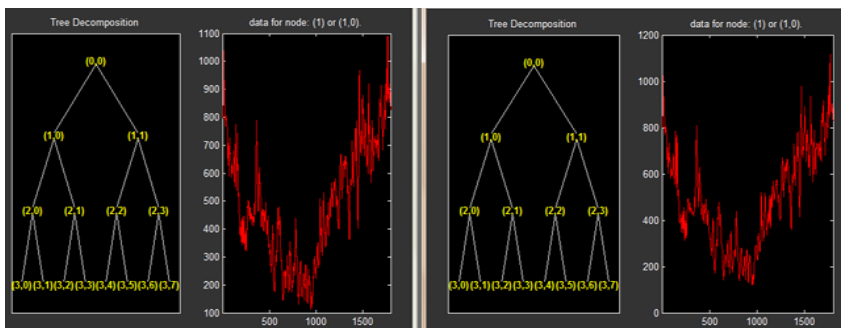

Figure 11. High frequency component compare before and after denoising

(Left is the ordinary signal, right is the denoising signal )
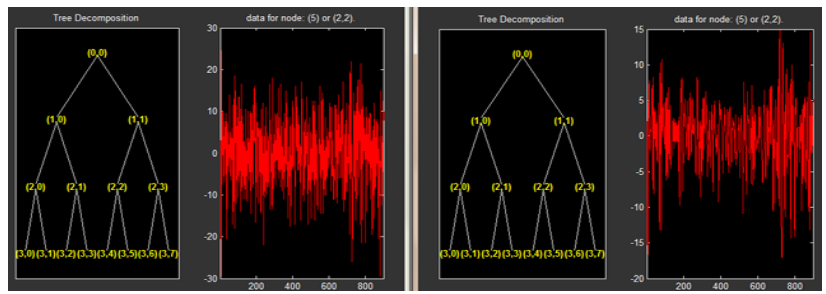

Figure 12. Low frequency component compare before and after denoising

(Left is the ordinary signal, right is the denoising signal )

We can learn: the high frequency part nearly be weakened but the low frequency part get smooth. So the low-frequency component of the output power can be filtered out through the appropriate method.

This conclusion is of great significance on smooth work for both wind turbine and wind farm.

\section{CONCLUSIONS}

To solve the fluctuation of wind turbine output power, this paper analyzes the output power based on the theory of 
the features of stationary sequence and wavelet transform. First, we judge the second-level output power is non-stationary through the mean and autocorrelation function aspects. Then we study the components and properties of the power based on the wavelet analysis. Through different analysis, we draw the conclusion that the main frequency components of this output power is 7 to $14 \mathrm{~Hz}$.We can get the different components from the wavelet decomposition .Wavelet filter can smooth the output power well. Only the low-frequency components can be filtered out.

\section{ACKNOWLEDGMENT}

The research is supported by the Shanghai Natural Science Foundation (No.14ZR1400700)

\section{REFERENCES}

[1] Karaki S H,Salim B A. Chedid R B .Probabilistic model of a two site wind energy conversion system[J].IEEE Trans on Energy Conversion,2002,17(4):530-536.

[2] Billinton R, Chowdhury A A. Incorporation of wind energy conversion systems in conventional generating capacity adequacy assessment[J]. IEE Proceeding-Generation, Transmission and Distribution, 1992, 139(1):47-56

[3] Li Shijie. The wind output power characteristically analysis. Journal of Hebei electrical power technology.2012.12

[4] Xiao Chuangying. Characteristic analysis of Gansu Jiuquan wind output power. Journal of Automation of electrical power system.2010.9

[5] Li Jiannan. Statistical modeling and study of large scale wind power output fluctuation of multi-scale. Journal of power protection and control system. 2012.10.

[6] Lin Jin. The model and analysis technology of wind power fluctuation simulation based on frequency domain.. Journal of Automation of electrical power system.2011.2

[7] Zhang Defeng. Matlab engineering application of wavelet analysis and. The defense industry press.2008.2 